\title{
Energy-based evaluation of seismic response of structures with passive and active systems
}

\author{
A. Yanik, U. Aldemir \& M. Bakioglu \\ Department of Civil Engineering, Istanbul Technical University, Turkey
}

\begin{abstract}
In recent years, increased performance requirements and the strong desire for better utilization of new materials and lower costs have motivated the development of new concepts for protecting structures. Recent practical applications and research findings show that the passive and active control techniques have the potential of protecting civil engineering structures from severe earthquakes and strong winds. In this paper, application potentials of passive and active control systems to earthquake excited structures are investigated in terms of energy distribution in the structure. Dynamic behavior of a three storey shear frame structure, incorporating passive and active control systems, subjected to El Centro, Erzincan and the simulated ground motions is investigated. The well-known Kanai-Tajimi power spectral density function is used to generate the simulated ground motion. Instantaneous optimal control algorithm which minimizes a performance index defined as a time dependent quadratic scalar functional instead of a quadratic integral functional and takes into account only the current state is used as an active control algorithm. A computer program which takes into account the nonlinear material property is developed for the dynamic analysis of the controlled and the uncontrolled structure. Nonlinear differential equation of the motion is solved by Wilson- $\theta$ numerical method. The numerical simulation results are presented in a comparative way for uncontrolled, passively controlled, actively controlled structures by graphical representations with hysteretic curves and the distributions of the damping energy, total kinetic energy, strain energy and seismic energy. An energy based evaluation of the example structure incorporating different combinations of passive and active systems is made.

Keywords: energy, structural control, earthquake, active control, passive control.
\end{abstract}




\section{Introduction}

The theoretical background of incorporating smart devices into structures was first given by Yao [1] in 1972. He stated in his paper that the control theories could be used in structural control problems. Structural control can be categorized as passive, active and hybrid control. In actively controlled system, structural responses are regulated by control forces based on a feedback system. Control forces are generated by external energy supply available in the system. External energy supply is not required in passive systems. Passive control devices transform the input seismic energy into different forms. Hybrid control systems incorporate both the active and passive devices. The history and the development of these systems are largely summarized in the work by Housner et al. [2]. Design of these systems with taking into account the energy and also energy based earthquake resistant design is still a growing research area. In this type of design the most important parameter is the amount of seismic energy input. As the amount of seismic energy input to structure is decreased or diminished by control devices, earthquake resistance of the structure increases. Some studies taking into account the energy of the structure in structural control problems are given in [3-8]. Actively controlled seismic excited structures was investigated with using optimal control theory in [9]. A study about a new control algorithm which is based on prediction of the near future earthquake excitations is given in [10]. A predictive semi active control method to suppress the earthquake response using a nonlinear semiactive damper was researched in [11]. A simple active control algorithm was proposed in [12].

The influence of the different control cases on the distribution of energy in a structure is studied in this paper. The material model is taken as bilinear elastoplastic. Three different cases of structural control are analysed and compared with each other and with uncontrolled structure. These cases are structure with base isolation, structure with base isolation and passive mass damper at the base of the building and structure with base isolation and active mass damper at the top of the building.

\section{Formulation of the problem}

The investigated shear building model is given for structure with base isolation, base isolation and passive mass damper at the base of the building and a base isolation and active mass damper at the top of the building respectively in fig. 1(a), (b), (c). The first two structures are the passively controlled structures while the last system can be classified as a hybrid control system.

For an inelastic shear-building with $n$ degrees of freedom under the one dimensional ground motion and control forces, the equation of the motion of the structure can be written in matrix form as

$$
\mathbf{M} \ddot{\mathbf{Y}}(t)+\mathbf{F}^{d}(t)+\mathbf{F}^{k}(t)=-\mathbf{M V} f(t)+\mathbf{L} \mathbf{U}(t)
$$




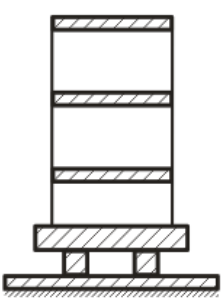

(a)

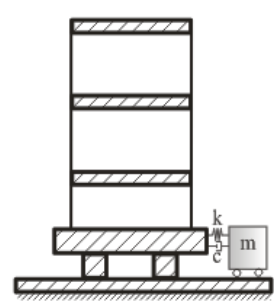

(b)

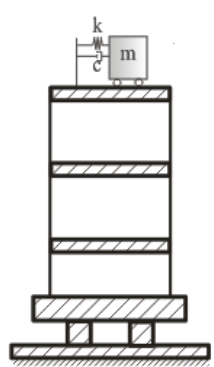

(c)

Figure 1: $\quad$ Structure with different control schemes.

where $\mathbf{Y}(t)=\left(y_{1}, y_{2}, \ldots ., y_{n}\right)^{\mathrm{T}}$ is the $n$-dimensional response vector denoting the relative displacement of the each storey unit with respect to the ground; $\mathbf{M}$ is the $(n \times n)$-dimensional constant mass matrix with diagonal elements $\left(m_{i}=\right.$ mass of $i$ th storey, $i=1,2, \ldots, n) ; \mathbf{F}^{d}(t)$ and $\mathbf{F}^{k}(t)$ are $n$-dimensional damping and stiffness force vectors respectively, $\mathbf{V}=(1, \ldots \ldots, 1)^{\mathrm{T}}$ is the $n$-dimensional vector; $\mathbf{L}$ is the $(n \times r)$-dimensional location matrix of $r$ controllers; $\mathbf{U}(t)$ is the $r$-dimensional active control force vector and scalar function $f(\mathrm{t})$ is the one-dimensional earthquake acceleration. For the uncontrolled and passively controlled case the second term in the right hand side of the equation becomes zero.

With introducing a $2 n$-dimensional state vector, $\mathbf{Z}(t)$, as follows:

$$
\mathbf{Z}(t)=\left[\begin{array}{c}
\mathbf{Y}(t) \\
\dot{\mathbf{Y}}(t)
\end{array}\right]
$$

the second-order matrix equation of motion can also be rewritten as a first order matrix equation with dimension $2 n$ in the following form:

$$
\dot{\mathbf{Z}}(t)=\mathbf{A Z}(t)+\mathbf{B} \mathbf{U}(t)+\mathbf{H} f(t) \quad \mathbf{Z}(0)=\mathbf{Z}_{0}
$$

where

$$
\mathbf{A}=\left[\begin{array}{cc}
\mathbf{0} & \mathbf{I} \\
-\mathbf{M}^{-1} \mathbf{K} & -\mathbf{M}^{-1} \mathbf{C}
\end{array}\right] \quad \mathbf{B}=\left[\begin{array}{c}
\mathbf{0} \\
\mathbf{M}^{-1} \mathbf{L}
\end{array}\right] \quad \mathbf{H}=\left[\begin{array}{c}
\mathbf{0} \\
-\mathbf{V}
\end{array}\right]
$$

To obtain the control force $\mathbf{U}(t)$ in eqn. (3), a control algorithm must be chosen. After determining the control force, eqn. (3) can be solved. In this study, instantaneous optimal control algorithm which minimizes a performance index defined as a time dependent quadratic scalar functional instead of a quadratic integral functional and takes into account only the current state is used.

\subsection{Instantaneous optimal control algorithm}

This algorithm is developed for getting over the difficulties because of not knowing the earthquake time history in the beginning (Yang et al. [13], Akhiev 
et al. [14]). Time dependent performance measure which will be minimized can be expressed as

$$
J=\mathbf{Z}^{\mathrm{T}}(t) \mathbf{Q} \mathbf{Z}(t)+\mathbf{U}^{\mathrm{T}}(t) \mathbf{R} \mathbf{U}(t) \rightarrow \min
$$

In eqn.(5) $\mathbf{Q}$ is a $(2 n \times 2 n)$-dimensional positive semidefinite symmetric weighting matrix and $\mathbf{R}$ is a $(r \times r)$-dimensional positive-definite symmetric weighting matrix. The values of $\mathbf{Q}$ and $\mathbf{R}$ matrices are assigned according to relative importance of the state variables and the control forces in the minimization procedure. If the elements of the matrix $\mathbf{Q}$ are chosen larger than the elements of $\mathbf{R}$, minimizing the response of the structure is more important than the control forces otherwise the minimization of the control forces are more important.

The basic equation of the problem is given in eqn. (3). Let the eigenvalues of $\mathbf{A}$ in eqn.(3) are distinct and $\mathbf{T}$ is the $(2 n \times 2 n)$-dimensional modal matrix which is constituted by the eigenvectors of $\mathbf{A}$. In this situation $\mathbf{Z}$ vector can be expressed as

$$
\mathbf{Z}=\mathbf{T X}(t)
$$

In eqn. (6), $\mathbf{X}(t)$ is the modal state vector. If we insert eqn. (6) into eqn. (3)

$$
\begin{gathered}
\dot{\mathbf{Z}}(t)=\mathbf{T} \dot{\mathbf{X}}(t)=\mathbf{A T X}(t)+\mathbf{B U}+\mathbf{H} f(t) \\
\dot{\mathbf{X}}(t)=\mathbf{T}^{-1} \mathbf{A T X}(t)+\mathbf{T}^{-1} \mathbf{B} \mathbf{U}+\mathbf{T}^{-1} \mathbf{H} f(t)
\end{gathered}
$$

$\mathbf{T}^{-\mathbf{1}} \mathbf{A T}$ term in eqn. (8) is the $\boldsymbol{\Lambda}$ spectral matrix of $\mathbf{A}$ matrix. The matrix $\boldsymbol{\Lambda}$ is a diagonal matrix of which diagonal element are eigenvalues of $\mathbf{A}$. By using $\boldsymbol{\Lambda}$ spectral matrix eqn. (8) can be expressed as

$$
\begin{aligned}
& \dot{\mathbf{X}}(t)=\mathbf{\Lambda} \mathbf{X}(t)+\mathbf{q}(t) \quad \mathbf{X}(0)=0 \\
& \mathbf{\Lambda}=\mathbf{T}^{-1} \mathbf{A} \mathbf{T} \quad \mathbf{q}(t)=\mathbf{T}^{-1} \mathbf{B} \mathbf{U}+\mathbf{T}^{-1} \mathbf{H} f(t)
\end{aligned}
$$

The solution of the differential equation in eqn. (9) can be written as

$$
\begin{gathered}
\mathbf{X}(t)=\mathbf{e}^{\Lambda t} \mathbf{X}(0)+\int_{0}^{t} \mathbf{e}^{\Lambda(t-\tau)} \mathbf{q}(\tau) d \tau=0+\int_{0}^{t} \mathbf{e}^{\Lambda(t-\tau)} \mathbf{q}(\tau) d \tau \\
\mathbf{X}(t)=\int_{0}^{t} \mathbf{e}^{\Lambda(t-\tau)} \mathbf{q}(\tau) d \tau+\int_{t-\Delta t}^{t} \mathbf{e}^{\Lambda(t-\tau)} \mathbf{q}(\tau) d \tau
\end{gathered}
$$

By using numerical integration(trapezoidal rule) for eqn. (12) the equation given below can be obtained

$$
\mathbf{X}(t) \approx \mathbf{e}^{\Lambda t} \mathbf{X}(t-\Delta t)+\frac{\Delta t}{2}\left[\mathbf{e}^{\Lambda \Delta t} \mathbf{q}(t-\Delta t)+\mathbf{q}(t)\right]
$$

In eqn. (10) $\mathbf{e}^{\Lambda \mathrm{t}}$ is a diagonal matrix and its diagonal elements are $\lambda_{\mathrm{i}} \Delta \mathrm{t}$ ( $\lambda$ ' are the eigenvalues of $\mathbf{A}$ matrix and subscript $i$ is from 1 to $2 \mathrm{n}$ ). By using eqn. (6) and $\mathbf{q}(\mathrm{t})$ from eqn. (10) $\mathbf{Z}$ can be expressed as 


$$
\begin{gathered}
\mathbf{Z}(t)=\mathbf{T D}(t-\Delta t)+\frac{\Delta t}{2}[\mathbf{B U}(t)+\mathbf{H} f(t)] \\
\mathbf{D}(t-\Delta t)=\exp (\Delta \Delta t) \mathbf{T}^{-1}\left\{\mathbf{Z}(t-\Delta t)+\frac{\Delta t}{2}[\mathbf{B} \mathbf{U}(t-\Delta t)+\mathbf{H} f(t-\Delta t)]\right\}
\end{gathered}
$$

Hamiltonian of the problem and the corresponding optimality conditions can be written as

$$
\begin{gathered}
H=\mathbf{Z}^{\mathrm{T}}(t) \mathbf{Q} \mathbf{Z}(t)+\mathbf{U}^{\mathrm{T}}(t) \mathbf{R} \mathbf{U}(t) \\
+\boldsymbol{\lambda}^{\mathrm{T}}(t)\left\{\mathbf{Z}(t)-\mathbf{T} \mathbf{D}(t-\Delta t)-\frac{\Delta t}{2}[\mathbf{B} \mathbf{U}(t)+\mathbf{H} f(t)]\right\} \\
\frac{\partial H}{\partial \mathbf{Z}^{*}}=0 \frac{\partial H}{\partial \mathbf{U}^{*}}=0 \frac{\partial H}{\partial \boldsymbol{\lambda}^{*}}=0
\end{gathered}
$$

By using these optimality conditions, the following equations are obtained,

$$
\begin{gathered}
2 \mathbf{Q Z} *+\lambda *(t)=0 \\
2 \mathbf{R U} *-\frac{\Delta t}{2} \mathbf{B}^{\mathrm{T}} \lambda *(t)=0 \\
\mathbf{Z}^{*}=\mathbf{T D} *(t-\Delta t)+\frac{\Delta t}{2}[\mathbf{B} \mathbf{U} *(t-\Delta t)+\mathbf{H} f(t-\Delta t)]
\end{gathered}
$$

For closed loop control, assuming that

$$
\lambda *(t)=\mathbf{P Z}(t)
$$

and substituting the eqn (21) into eqn (18), $\mathbf{P}=\mathbf{- 2 Q}$ is obtained. Then from eqs (19) and (20), the following equations are derived;

$$
\begin{gathered}
\mathbf{U}^{*}=-\frac{\Delta t}{2} \mathbf{R}^{-1} \mathbf{B}^{\mathrm{T}} \mathbf{Q Z} *(t) \\
\mathbf{Z}^{*}=\left[\mathbf{I}+\frac{(\Delta t)^{2}}{4} \mathbf{B R}^{-1} \mathbf{B}^{\mathrm{T}} \mathbf{Q}\right]^{-1}\left[\mathbf{T D}(t-\Delta t)+\frac{\Delta t}{2} \mathbf{H} f(t)\right]
\end{gathered}
$$

\section{Energy equations}

If eqn. (1) is multiplied with the transpose of velocity vector $\dot{\mathbf{Y}}^{\mathrm{T}}(\mathrm{t})$ and integrated in the interval of $(0-t)$, the energy equations will be obtained and expressed as 


$$
\begin{gathered}
\int_{0}^{t} \dot{\mathbf{Y}}^{\mathrm{T}}(\tau) \mathbf{M} \ddot{\mathbf{Y}}(\tau) d \tau+\int_{0}^{t} \dot{\mathbf{Y}}^{\mathrm{T}}(\tau) \mathbf{F}^{d}(\tau) d \tau+\int_{0}^{t} \dot{\mathbf{Y}}^{\mathrm{T}}(\tau) \mathbf{F}^{k}(\tau) d \tau \\
=-\int_{0}^{t} \dot{\mathbf{Y}}^{\mathrm{T}}(\tau) \mathbf{M V} \ddot{x}_{0} d \tau+\int_{0}^{t} \dot{\mathbf{Y}}^{\mathrm{T}}(\tau) \mathbf{L} \mathbf{U} d \tau
\end{gathered}
$$

The first term in eqn. $(24)$ is called kinetic energy $\left(E_{k i n}\right)$ and can be written as

$$
E_{k i n}=\int_{0}^{t} \dot{\mathbf{Y}}^{\mathrm{T}}(\tau) \mathbf{M} \ddot{\mathbf{Y}}(\tau) d \tau=\int_{0}^{t}\left[\dot{\mathbf{Y}}^{\mathrm{T}}(\tau) \mathbf{M} \dot{\mathbf{Y}}(\tau)\right] d \tau=\frac{1}{2} \dot{\mathbf{Y}}^{\mathrm{T}}(t) \mathbf{M} \dot{\mathbf{Y}}(t)
$$

$E_{k i n}$ is sum of the kinetic energy of all masses with respect to the ground. In eqn. (24) the second term is equal to the damping energy of the system, damping energy $E_{\text {damp }}$ can be expressed as

$$
E_{d a m p}=\int_{0}^{t} \dot{\mathbf{Y}}^{\mathrm{T}}(\tau) \mathbf{F}^{k}(\tau) d \tau
$$

The third term in eqn. (25) is the strain energy $E_{\text {str }}$ and can be written as

$$
E_{s t r}=\int_{0}^{t} \dot{\mathbf{Y}}^{\mathrm{T}}(\tau) \mathbf{F}^{k}(\tau) d \tau
$$

If the system is linear elastic $\mathbf{F}^{k}=\mathbf{K Y}$ in which $\mathbf{K}$ is the stiffness matrix, the second term in eqn. (25) for linear elastic case, can be expressed as

$$
E_{\text {str }}^{\text {elas }}=\int_{0}^{t} \dot{\mathbf{Y}}^{\mathrm{T}}(\tau) \mathbf{K Y}(\tau) d \tau=\frac{1}{2} \mathbf{Y}^{\mathrm{T}}(t) \mathbf{K Y}(t)
$$

Eqn. (28) is valid if the system is totally elastic. If the strains of the system exceed the elastic limit, strain energy can be divided to two types, one of which is returned elastic energy and the other is unreturned hysteretic energy. For this case total strain energy can be expressed as total of two types of energy

$$
E_{s t r}=E_{s t r}^{\text {elas }}+E_{s t r}^{\text {his }}
$$

The first term in the right hand side of eqn. (24) is the earthquake energy of the structure $E_{\text {earth }}$ and the last term is the control energy $E_{c o n}$, these energies can be expressed as

$$
E_{\text {earth }}=-\int_{0}^{t} \dot{\mathbf{Y}}^{\mathrm{T}}(\tau) \mathbf{M V} \ddot{x}_{0} d \tau \quad E_{\text {con }}=\int_{0}^{t} \dot{\mathbf{Y}}^{\mathrm{T}}(\tau) \mathbf{L} \mathbf{U} d \tau
$$

If we add eqn (31) to the left side of the eqn.(24) it will have a negative sign in front of it. Although it has a negative sign it is positive because control forces generally depends negatively on displacement and velocity response so this makes control energy positive. After defining this, energy equilibrium can be expressed as

$$
E_{\text {kin }}+E_{\text {damp }}+E_{s t r}^{\text {elas }}+E_{\text {str }}^{\text {his }}+E_{\text {con }}=E_{\text {earth }}
$$




\section{Numerical example}

As an example problem a three-storey shear building with damping is considered under three different earthquake motions which are El-Centro, Erzincan and simulated ground motion. For simulated ground motion Kanai-Tajimi power spectral density function is used. More information about this function can be found Shinozuka and Deodatis [15]. All these three earthquakes can be classified as strong motions, simulated ground motion has maximum acceleration of $0.33 \mathrm{~g}$. Acceleration time histories of El Centro, Erzincan and synthetic earthquakes are given in fig. (2). The columns of the building are assumed as massless and the mass of the structure is concentrated in floor level as lumped mass model. Each storey has the same mass, stiffness and damping parameters. As control elements base isolation and mass damper are chosen. Mass, stiffness and damping parameters of the base isolation are $m_{b}=100$ tons, $k_{b}=2527 \times 10^{3}$ $\mathrm{kN} / \mathrm{m}$ and $c_{b}=63 \mathrm{kNs} / \mathrm{m}$ respectively. For passive and active mass damper the parameters are $m_{d}=36.3$ tons, $k_{d}=1173 \mathrm{kN} / \mathrm{m}, c_{d}=31 \mathrm{kNs} / \mathrm{m}$. Mass, stiffness and damping matrix of the structure for uncontrolled case are as follows:

$$
\begin{gathered}
\mathbf{M}=\left[\begin{array}{ccc}
100 & 0 & 0 \\
0 & 100 & 0 \\
0 & 0 & 100
\end{array}\right] \text { ton, } \mathbf{C}=\left[\begin{array}{ccc}
251.32 & -125.66 & 0 \\
-125.66 & 251.32 & -125.66 \\
0 & -125.66 & 125.66
\end{array}\right] \mathrm{kNs} / \mathrm{m} \\
\mathbf{K}=\left[\begin{array}{ccc}
31582.734 & -15791.34 & 0 \\
-15791.34 & 31582.734 & -15791.34 \\
0 & -15791.34 & 15791.34
\end{array}\right] \mathrm{kN} / \mathrm{m}
\end{gathered}
$$

Energy distributions and force-displacement responses of the structure are investigated for four cases which are uncontrolled case (1), structure with base isolation (2), structure with base isolation and passive mass damper(3), and structure with base isolation and active mass damper (4). For active control case weighting matrix $\mathbf{R}(1 \times 1)$ is chosen as $10^{-3}$, a scalar number because only a single active mass damper is in the system. The other weighting matrix $\mathbf{Q}$ is chosen as $(10 \times 10)$ dimensional diagonal matrix of which diagonal elements are equal to 1000 .

Peak values of the relative displacements for the storeys and supplemental elements are given in Table 1. It can be seen from the table that for all cases the largest reduction in the storey displacements have been obtained for the hybrid system which consists of an active mass damper and base isolation. Active mass damper has also reduced the displacement of the base isolation. Adding passive mass damper to the structure with base isolation has also reduced the storey displacements for the cases of El Centro and Synthetic earthquakes. But, for Erzincan earthquake the displacements are approximately the same. This can be because of the stronger effect of Erzincan earthquake than the other ones. Moreover implementing base isolation to the uncontrolled structure has reduced the displacements. 

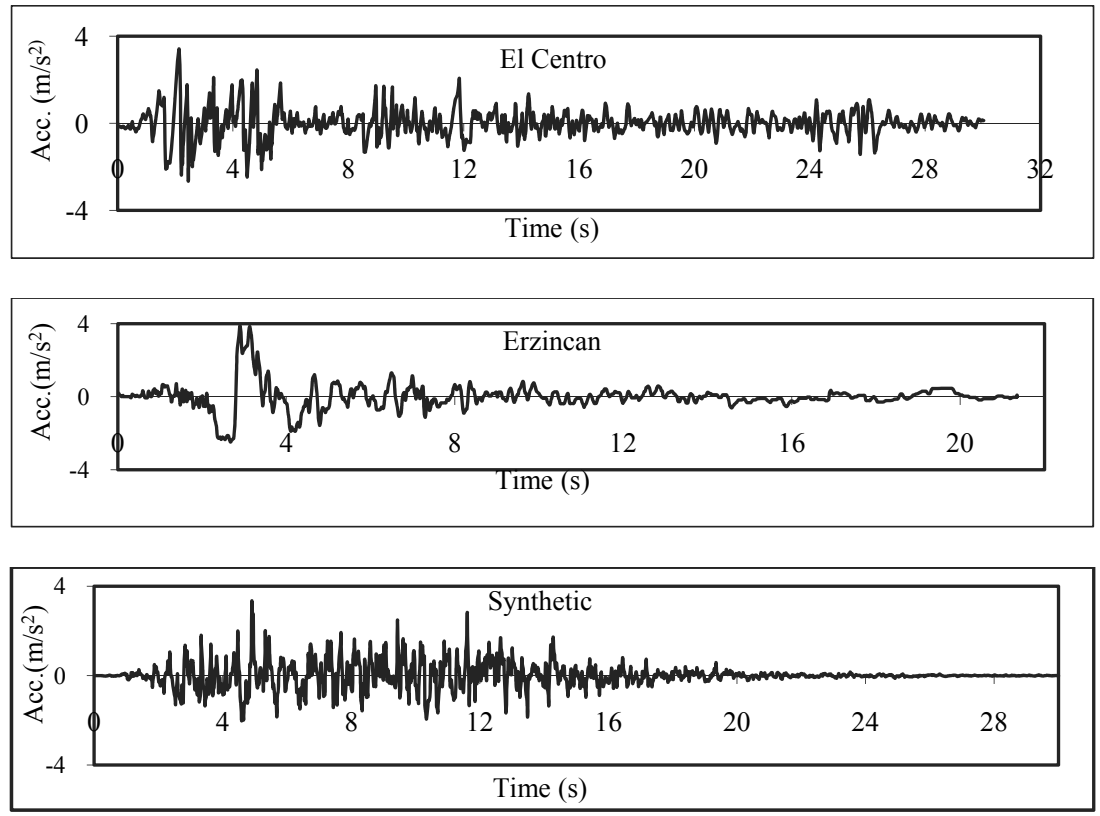

Figure 2: $\quad$ Earthquake time history.

Table 1: $\quad$ Maximum storey displacements (m).

\begin{tabular}{lccccc}
\hline Earthquake & $\begin{array}{c}\text { Storey } \\
\text { No. }\end{array}$ & & \multicolumn{3}{c}{ Control Cases } \\
& & 1 & 2 & 3 & 4 \\
\hline \multirow{5}{*}{ El Centro } & Mass Damper & - & - & 0.0649 & 0.0516 \\
& Base Isolation & - & 0.2039 & 0.2144 & 0.1868 \\
& 1 & 0.0670 & 0.0846 & 0.0592 & 0.0552 \\
& 2 & 0.0371 & 0.0198 & 0.0180 & 0.0177 \\
& 3 & 0.0188 & 0.0109 & 0.0097 & 0.0109 \\
\hline \multirow{5}{*}{ Erzincan } & Mass Damper & - & - & 0.1401 & 0.1491 \\
& Base Isolation & - & 0.3306 & 0.3649 & 0.3410 \\
& 1 & 0.2801 & 0.2700 & 0.3018 & 0.2391 \\
& 2 & 0.1510 & 0.1713 & 0.2031 & 0.1527 \\
& 3 & 0.0229 & 0.0236 & 0.0267 & 0.0226 \\
\hline \multirow{5}{*}{ Synthetic } & Mass Damper & - & - & 0.0315 & 0.0637 \\
& Base Isolation & - & 0.1641 & 0.1714 & 0.1695 \\
& 1 & 0.0387 & 0.0200 & 0.0191 & 0.0245 \\
& 2 & 0.0291 & 0.0141 & 0.0131 & 0.0147 \\
& 3 & 0.0176 & 0.0077 & 0.0068 & 0.0088 \\
\hline
\end{tabular}

The hysteretic curves for the second storey of the structure under El Centro earthquake are given in fig. 3 for all cases. In fig. 3, the numbers (1) to (4) represent the control cases defined above. Implementing base isolation to the system makes the structural behavior elastic for El Centro earthquake. 


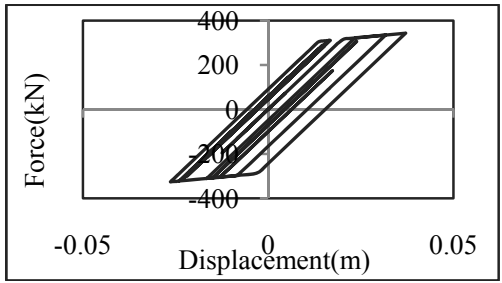

(1)

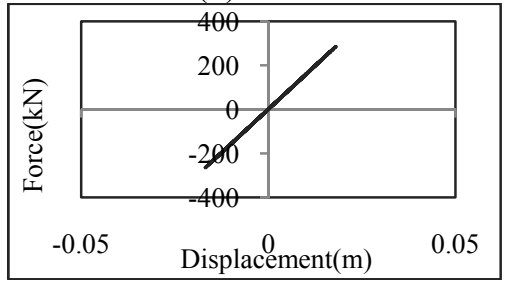

(3)

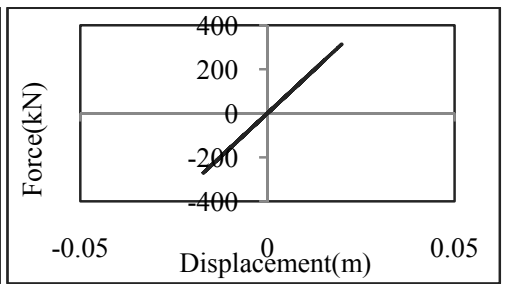

(2)

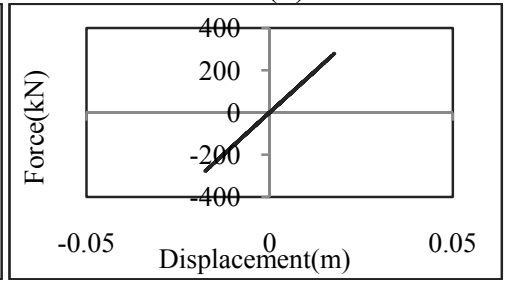

(4)

Figure 3: Hysteretic curve for El Centro earthquake.

It is known that the sum of kinetic energy, damping energy, strain energy and control energy is equal to earthquake energy. For passive control cases control energy is zero for all the earthquake duration. In addition; the increase in the control forces result in the increase in the control energy consumption. For this reason appropriate numerical values are assigned to the elements of $\mathbf{Q}$ and $\mathbf{R}$ matrices. For the uncontrolled, passive and active control cases, energy distributions are given in fig. 4 for synthetic earthquake. It can be seen from fig. 4 that for uncontrolled case the strain energy curve oscillated between the values of

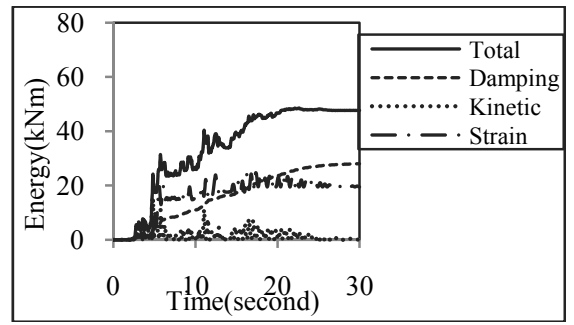

(1)

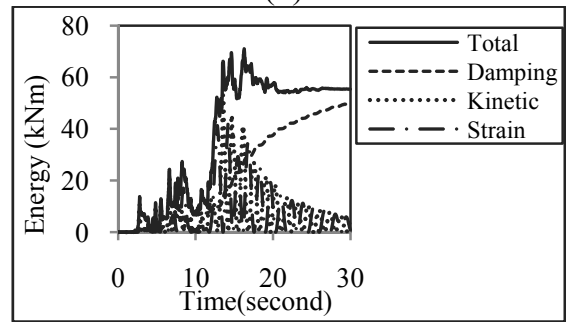

(3)

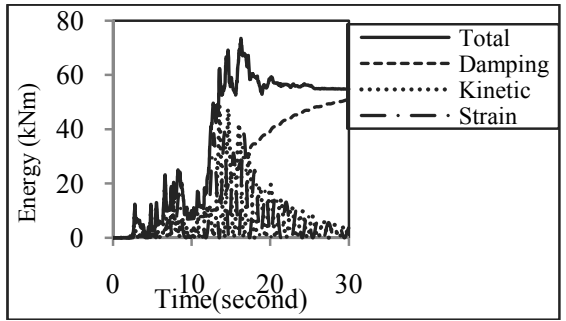

(2)

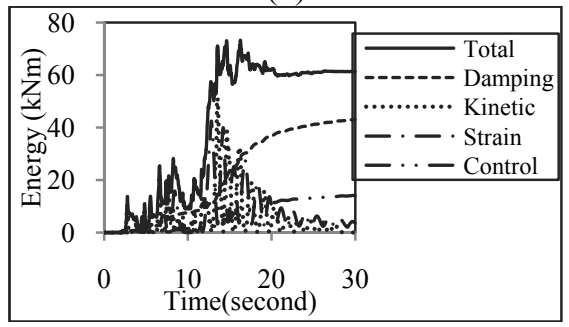

(4)

Figure 4: $\quad$ Seismic energy distributions for synthetic earthquake. 
$14-21(\mathrm{kNm})$, this is because of the plastic strains of the structure. Implementing passive or active devices to the structure makes the structural behavior elastic. For active control case maximum damping energy has decreased nearly \%14 and the maximum control energy for providing control forces is $13.95 \mathrm{kNm}$.

The energy distributions of the structure subjected to El Centro earthquake are given in fig. 5. For El Centro earthquake, the largest reduction in the strain energy has been obtained for the hybrid system, while the maximum control energy for this system is $29.11 \mathrm{kNm}$. In fig. 5 it can be seen that strain energy oscillates between $70 \mathrm{kNm}$ and $92 \mathrm{kNm}$. This is because of the plastical behavior of the structure under El Centro earthquake. The energy distributions for Erzincan earthquake is given in fig.6.

Under Erzincan earthquake for active control case, the maximum control energy is $38.15 \mathrm{kNm}$. For three different earthquakes, adding base isolation and mass damper to structure has increased the total energy consumption of the structure.

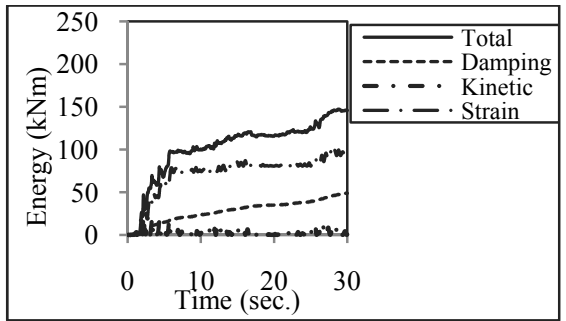

(1)

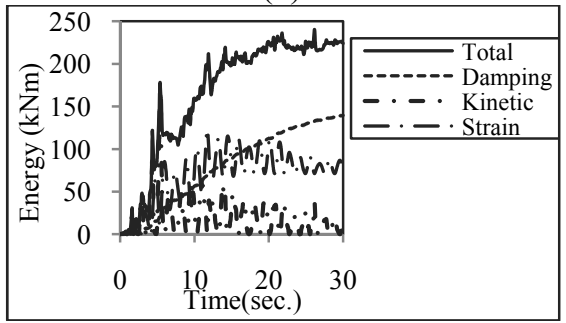

(3)

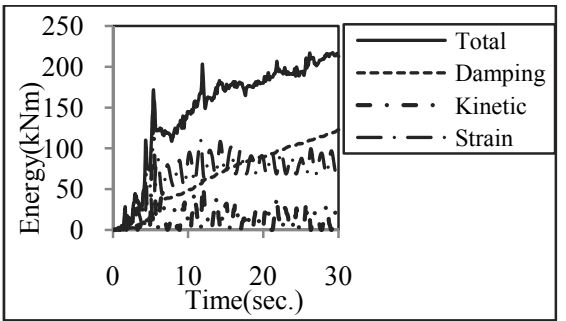

(2)

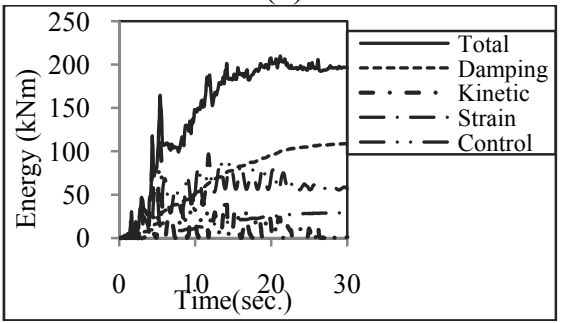

(4)

Figure 5: $\quad$ Seismic energy distributions for El Centro earthquake.

\section{Conclusion}

In this study dynamic behavior and the energy distributions in structures with passive and active control elements are investigated. It has been found that implementing control devices to structures reduces significantly the displacement responses. Applying passive or active control to uncontrolled structure, increases 


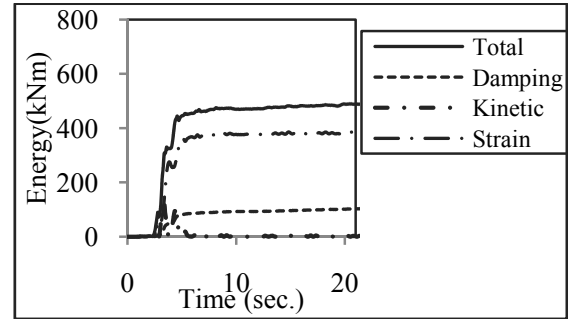

(1)

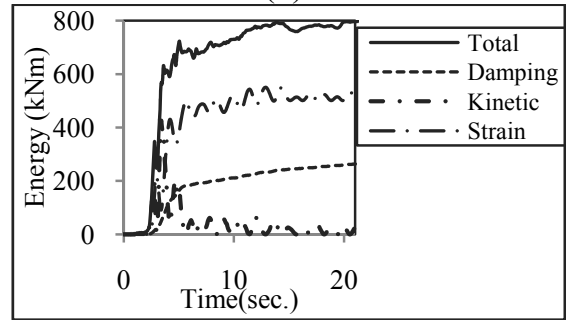

(3)

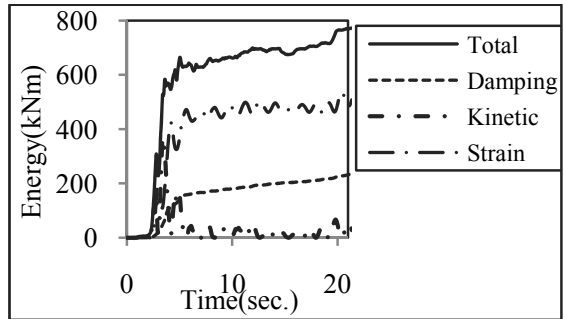

(2)

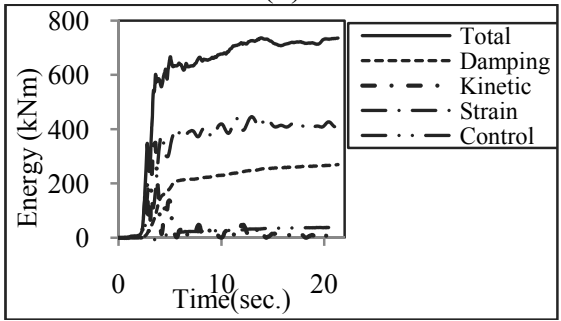

(4)

Figure 6: Seismic energy distributions for Erzincan earthquake.

the total energy consumption. Structure with base isolation and passive mass damper and structure with base isolation and active mass damper at the top of the building are the most effective control systems. Implementing two different control elements to a structure may be more feasible than a single active or passive device.

\section{References}

[1] Yao, J.T.P., Concept of structural control. Journal of Structural Division ASCE, 98, pp.1567-1574, 1972.

[2] Housner, G.W., Bergman, L.A., Caughey, T.K., Chassiakos, A.G., Claus, R.O., Masri, S.F., Skelton, R.E., Soong, T.T., Spencer, B.F. \& Yao, J.T.P., Structural Control: Past, Present, and Future. Journal of Engineering Mechanics, ASCE, 123(2), pp.897-958, 1997.

[3] Wong, K.K.F. \& Wang, Y. Probabilistic structural damage assessment and control based on energy approach. The Structural Design of Tall Buildings, 10,pp. 283-308, 2001.

[4] Wong, K.K.F. \& Zhao, D., Effectiveness of inelastic structural control based on elastic displacement and energy. Structural Control and Health Monitoring, 12,pp. 47-64, 2005.

[5] Wong, K.K.F. \& Yang, R., Effectiveness of structural control based on control energy perspectives. Earthquake Engineering and Structural Dynamics, 30, pp.1747-1768, 2001. 
[6] Wong, K.K.F. \& Pang, M., "Energy density spectra in actively controlled inelastic structures-theory". Structural Control and Health Monitoring, 14, pp.261-278, 2007.

[7] Wong, K.K.F. \& Pang, M., Energy density spectra in actively controlled inelastic structures-application. Structural Control and Health Monitoring, pp.14, 279-300, 2007.

[8] Lu, L.Y., Lin, G.L. \& Lin,C.,C., Absolute-energy-based active control strategies for linear seismic isolation systems. Structural Control and Health Monitoring, DOI:10.1002/stc.373 2010.

[9] Aldemir, U., Bakioglu, M. \& Akhiev S.S.,Optimal control of linear buildings under seismic excitations. Earthquake Engineering and Structural Dynamics, 30,pp.835-851, 2001.

[10] Aldemir, U. \& Bakioglu, M., Active structural control based on prediction and degree of stability. Journal of Sound and Vibration, 247(4) 561-576, 2001.

[11] Aldemir, U., Predictive suboptimal semiactive control of earthquake response. Structural Control and Health Monitoring, 17 , 654-674, 2010.

[12] Aldemir, U., A simple active control algorithm for earthquake excited structures. Computer-Aided Civil and Infrastructure Engineering, 25, 218$225,2010$.

[13] Yang, J.N., Akbarpour, A. \& Ghaemmaghami, P., New Optimal Control Algoritms for Structural Control. J.Eng. Mech. ASME, 113,9 pp. 13691386, 1987.

[14] Akhiev, S. S., Aldemir, U. \& Bakioglu, M., Multipoint Instantaneous Optimal Control of Structures. Computers and Structures, 80, pp.909-917, 2002.

[15] Shinozuka, M. \& Deodatis, G., Simulasyon of Stochastic Processes by Spectral Representation Appl. Mec. Rev. 44(4), pp.191-203, 1991. 